\title{
Transition between SU(4) and SU(2) Kondo effect
}

\author{
L. Tosi ${ }^{\text {a }}$ P. Roura-Bas ${ }^{b}$ A. A. Aligia ${ }^{a}$ \\ ${ }^{a}$ Centro Atómico Bariloche and Instituto Balseiro, Comisión Nacional de Energía \\ Atómica, 8400 Bariloche, Argentina \\ ${ }^{\mathrm{b}}$ Dpto de Física, Centro Atómico Constituyentes, Comisión Nacional de Energía \\ Atómica, Buenos Aires, Argentina
}

\begin{abstract}
Motivated by experiments in nanoscopic systems, we study a generalized Anderson, which consists of two spin degenerate doublets hybridized to a singlet by promotion of an electron to two conduction bands, as a function of the energy separation $\delta$ between both doublets. For $\delta=0$ or very large, the model is equivalent to a one-level $\mathrm{SU}(\mathrm{N})$ Anderson model, with $\mathrm{N}=4$ and 2 respectively. We study the evolution of the spectral density for both doublets $\left(\rho_{1 \sigma}(\omega)\right.$ and $\left.\rho_{2 \sigma}(\omega)\right)$ and their width in the Kondo limit as $\delta$ is varied, using the non-crossing approximation (NCA). As $\delta$ increases, the peak at the Fermi energy in the spectral density (Kondo peak) splits and the density of the doublet of higher energy $\rho_{2 \sigma}(\omega)$ shifts above the Ferrmi energy. The Kondo temperature $T_{K}$ (determined by the half width at half maximum of the Kondo peak in density of the doublet of lower energy $\rho_{1 \sigma}(\omega)$ ) decreases dramatically. The variation of $T_{K}$ with $\delta$ is reproduced by a simple variational calculation.
\end{abstract}

Key words: Anderson model, spectral density, Kondo temperature, non-crossing approximation

PACS: $75.20 . \mathrm{Hr}, 75.10 . \mathrm{Jm}$

\section{Introduction}

The Kondo effect, found originally for systems with magnetic impurities in metals is now present in a variety of nanoscopic systems, including semiconducting quantum dots [1], magnetic adatoms on surfaces [1,2,3] and carbon nanotubes [4]. In the latter, in addition to the spin Kramers degeneracy, there

* Tel: 54-11 67727102; FAX: 54-11 67727121; roura@tandar.cnea.gov.ar 
is in addition orbital degeneracy due to the "pseudospin" degree of freedom related with the particular band structure of graphene. This leads to an SU(4) Kondo effect which has been observed experimentally [5,6] and also discussed theoretically $[7,8,9]$. In particular, Lim et al. have studied the spectral density when the $\mathrm{SU}(4)$ symmetry is reduced to $\mathrm{SU}(2)$, mainly by a change in the tunneling matrix elements $[7,9]$.

Our main motivation in the problem arises from interference phenomena in systems of quantum dots $[10,11,12]$ or molecules $[11,13,14]$. For example depressions in the integrated conductance through a ring described by the Hubbard or $t-J$ model, pierced by an Aharonov-Bohm magnetic flux, related with spin-charge separation $[10,11]$, are due to a partial destructive interference when the energy of two doublets cross [11]. Similar interference effects were predicted in molecular transistors $[13,14]$. The effective Hamiltonian near the crossing is discussed in the next section.

To our knowlege, the conductance in these systems has so far been calculated using approximate expressions or a slave-boson formalism [12], which is valid only for very low temperatures and applied bias voltages. This work is a step towards a more quantitative theory to describe the transport through similar systems, treating the effective Hamiltonian within the non-crossing approximation (NCA) $[15,16]$. Work is in progress to deal with the non-equilibrium situation, which is necessary within our formalism to calculate the current.

In this paper, we report on our study of the spectral density of the model as a function of the splitting $\delta$ between both doublets in the Kondo regime. We also calculate the dependence of the Kondo temperature $T_{K}$ with $\delta$ and analyze the validity of the Friedel sum rule [17].

\section{Model}

We start from a model in which two doublets of an interacting system are hybridized with a singlet by promotion of an electron to two conducting leads. This is the low-energy effective Hamiltonian for several systems with partial destructive interference, such as Aharonov-Bohm rings [11] or aromatic molecules $[13,14]$ connected to conducting leads. The Hamiltonian can be written as $[11]$

$$
\begin{aligned}
H= & E_{s}|0\rangle\left\langle 0\left|+\sum_{i \sigma} E_{i}\right| i \sigma\right\rangle\langle i \sigma|+\sum_{\nu k \sigma} \epsilon_{\nu k} c_{\nu k \sigma}^{\dagger} c_{\nu k \sigma} \\
& +\sum_{i \nu k \sigma}\left(V_{i \nu}|i \sigma\rangle\langle 0| c_{\nu k \sigma}+\text { H.c. }\right)
\end{aligned}
$$


where the singlet $|0\rangle$ and the two doublets $|i \sigma\rangle(i=1,2 ; \sigma=\uparrow$ or $\downarrow)$ denote the localized states (representing for example the low-energy states of an isolated

molecule), $c_{\nu k \sigma}^{\dagger}$ create conduction states in the left $(\nu=L)$ or right $(\nu=R)$ lead, and $V_{i \nu}$ describe the four hopping elements between the two leads and both doublets, assumed independent of $k$.

Changing the phase of the conduction states and the relative phase between both doublets, three among the four $V_{i \nu}$ can be made real and positive. The phase $\phi$ of the remaining hopping $|V| e^{i \phi}$, depends on the particular system and its symmetry. For example in molecules with rotational symmetry $\phi=$ $\left(K_{1}-K_{2}\right) l$, where $l$ is the distance between the sites connected to the left and right leads, and $K_{i}$ is the wave vector of the state $|i \sigma\rangle$, which can be modified with an applied magnetic flux [11]. In absence of magnetic flux and when the relevant states have wave vector $K_{i}= \pm \pi / 2$, as in rings with a number of atoms multiple of four, $\phi=\pi$ and there is complete destructive interference in transport $[11,14]$.

In the following we will assume this case, with states 1 and 2 related by symmetry implying $\left|V_{1 \nu}\right|=\left|V_{2 \nu}\right|$. We further assume symmetric leads, $\epsilon_{L k}=$ $\epsilon_{R k}=\epsilon_{k},\left|V_{i L}\right|=\left|V_{i R}\right|$. Then, without loss of generality we can take $V_{1 L}=$ $V_{1 R}=V_{2 L}=V>0, V_{2 R}=-V$, and $E_{2} \geq E_{1}$.

For these parameters, changing basis $c_{1 k \sigma}^{\dagger}=\left(c_{L k \sigma}^{\dagger}+c_{R k \sigma}^{\dagger}\right) / \sqrt{2}, c_{2 k \sigma}^{\dagger}=\left(c_{L k \sigma}^{\dagger}-\right.$ $\left.c_{R k \sigma}^{\dagger}\right) / \sqrt{2}$, the Hamiltonian takes the form of an SU(4) Anderson model with a "field" $\delta=E_{2}-E_{1}$ and on-site hybridization $V^{\prime}=\sqrt{2} V$

$$
\begin{aligned}
H= & E_{s}|0\rangle\left\langle 0\left|+\sum_{i \sigma} E_{i}\right| i \sigma\right\rangle\langle i \sigma|+\sum_{i k \sigma} \epsilon_{k} c_{i k \sigma}^{\dagger} c_{i k \sigma} \\
& +V^{\prime} \sum_{i k \sigma}\left(|i \sigma\rangle\langle 0| c_{i k \sigma}+\text { H.c. }\right) .
\end{aligned}
$$

Interchanging the doublet index ( 1 or 2 ) with the spin index $\sigma$, one realizes that this model also describes transport trough a carbon nanotube with electron density depleated at two points (so as to created an SU(4) quantum dot in the middle) under a real applied magnetic field.

\section{Spectral density}

In Fig. 1 we present numerical results for the spectral density $\rho_{i \sigma}$ of the SU(4) Anderson model $\left(E_{1}=E_{2}\right)$ in the Kondo regime $\epsilon_{F}-E_{i} \gg \Delta$, where the hybridization function $\Delta=\pi \sum_{k}\left(V^{\prime}\right)^{2} \delta\left(\omega-\epsilon_{k}\right)$, assumed independent of energy. We set $\Gamma=2 \Delta=1$ as the unit of energy. We also assume a conduction band 


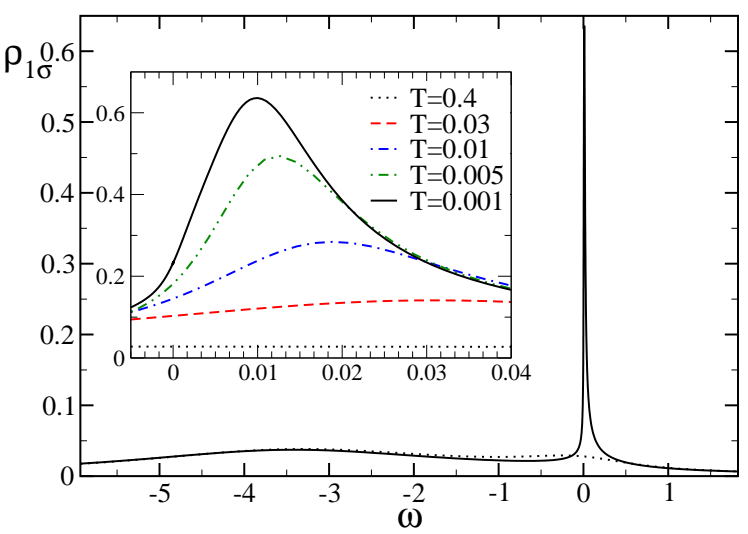

Fig. 1. Spectral density as a function of energy for different temperatures. The inset shows a detail near the Fermi energy. Parameters are $\Delta=0.5 . D=10$, $E_{1}=E_{2}=-4$. The lowest temperature is $T=10^{-3}=0.076 T_{K}$.

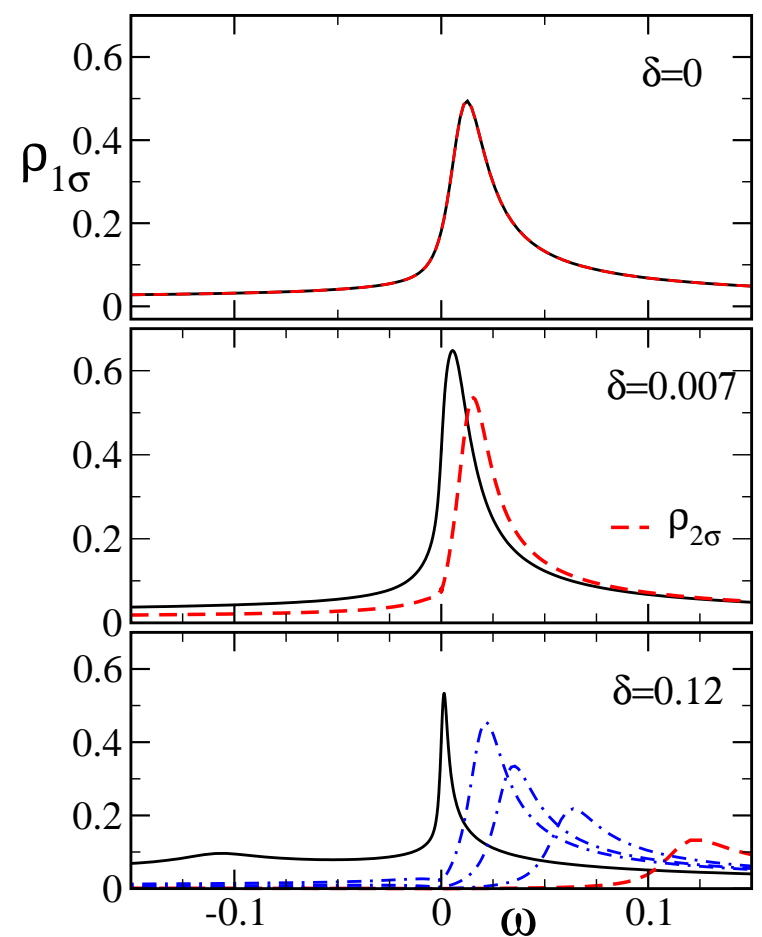

Fig. 2. Spectral densities for levels 1 (full line) and 2 (dashed line) as a function of energy for different values of $E_{2}=E_{1}+\delta$ and $T=10^{-3}$. Other parameters as in Fig. 1. Dot-dashed line corresponds to $\rho_{2 \sigma}$ for $\delta=0.015,0.3$ and 0.6.

symmetric around the Fermi level $\epsilon_{F}=0$, of half width $D=10$.

The spectral density shows a broad charge transfer peak near $E_{1}$. For temperatures below a characteristic energy scale $T_{K}$ (defined below), $\rho_{i \sigma}$ develops a narrow peak around the Fermi level. In contrast to the better known onelevel $\mathrm{SU}(2)$ case, this peak is displaced towards positive energies and is much broader, as discussed in Section 5 . 
The evolution of the spectral densities as $E_{2}$ is displaced to larger energies, breaking the SU(4) symmetry is shown in Fig. 2. The peak near the Fermi energy of $\rho_{2 \sigma}(\omega)$ is displaced towards positive energies (near $\delta=E_{2}-E_{1}$ ). In contrast, the corresponding peak in $\rho_{1 \sigma}(\omega)$ narrows significantly and displaces towards the Fermi energy. This implies that the Kondo temperature $T_{K}$ defined as the half width at half maximum of this peak, also decreases strongly. The evolution of $T_{K}$ with $\delta$ is discussed in the Section 5.

In addition $\rho_{1 \sigma}$ develops a broad peak near energy $-\delta$ which becomes visible when $\delta$ becomes greater than $T_{K}$.

\section{Friedel sum rule}

The Anderson model studied has a Fermi liquid ground state which satisfies well known relationships at zero temperature. One of them is the Friedel sum rule which relates the spectral density at the Fermi level for each "pseudospin" channel with the occupation of that channel [17]. For the simplest case of a constant density of conduction states, this rule reads

$$
\rho_{i \sigma}\left(\epsilon_{F}\right)=\frac{1}{\pi \Delta} \sin ^{2}\left(\pi n_{i \sigma}\right),
$$

where $n_{i \sigma}=\langle\mid i \sigma\rangle\langle i \sigma \mid\rangle$.

This is an exact relationship for a Fermi liquid, which is not necessarily satisfied by approximations. In particular, it is known that at very low temperatures, the NCA has a tendency to develop spurious spikes in $\rho_{i \sigma}(\omega)$ at the Fermi energy, while thermodynamic properties, such as expectation values are accurately reproduced $[15,16]$.

In Fig. 3, we compare both members of Eq. (3) for the lowest lying doublet, at a temperature $T=0.1 T_{K}[18]$ low enough so that no further increase in $\rho_{i \sigma}\left(\epsilon_{F}\right)$ takes place as the temperature is lowered (according to physical expectations and Eq. (3)), but high enough to prevent the presence of spurious spikes. The disagreement lies below $20 \%$. The agreement improves as the parameters are moved deeper in the Kondo regime $\epsilon_{F}-E_{1} \gg \Delta$. Thus, while the spectral density at zero temperature is not well represented by the NCA results at $T=0$, one can take the values at $T=0.1 T_{K}$ as a reasonable description of the correct $T=0$ ones. This statement is supported by the comparison of results obtained by NCA and numerical renormalization group for the onelevel $\mathrm{SU}(2)$ case [19]. 


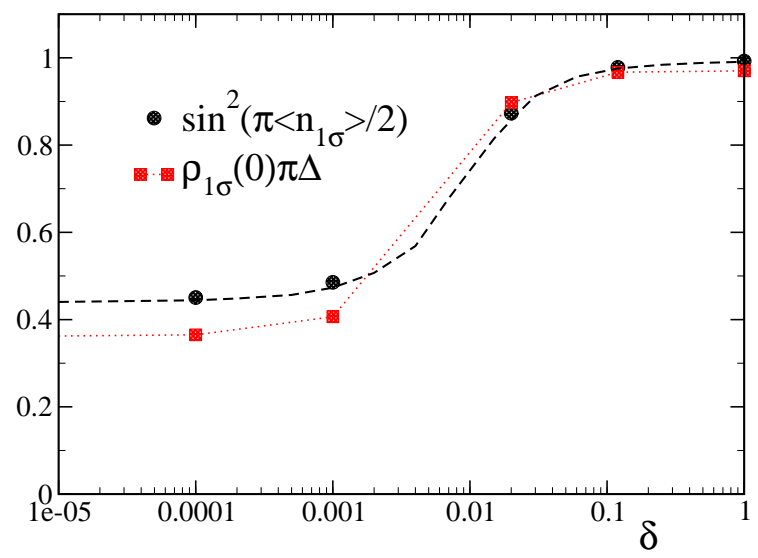

Fig. 3. Squares: rescaled spectral density of the lowest lying level at the Fermi energy as a function of $\delta$. Circles: corresponding (more accurate) result given by Eq. (3). Lines are guides to the eye. Parameters are $T=0.1 T_{K}$ and the rest as in Fig. 1.

\section{Kondo temperature}

From the half width at half maximum of the peak nearest to the Fermi energy of the spectral density of the lowest level $\left(\rho_{1 \sigma}(\omega)\right)$, we have calculated the Kondo temperature of the system $T_{K}$ for several values of $\delta$. This requires to solve the self-consistent NCA equations up to low enough temperatures (about $0.1 T_{K}$ as discussed above) so that the height of the peak does not increase significantly with further lowering of the temperature [18]. Fortunately, the result is not very sensitive to the ratio $T / T_{K}$.

The results are shown in Fig. 4 and compared with Eq. (5) obtained from a variational calculation as explained below. We see that except for an overall multiplicative factor, the agreement is quite good, in spite of the fact that $T_{K}$ changes by nearly two orders of magnitude.

To provide an independent estimate of $T_{K}$, we have calculated the stabilization energy of the following variational wave function

$$
|\psi\rangle=\alpha|s\rangle+\sum_{i k \sigma} \beta_{i k}\left(|i \sigma\rangle\langle 0| c_{i k \sigma}\right)|s\rangle
$$

where $|s\rangle$ is the many-body singlet state with the filled Fermi sea of conduction electrons and the state $|0\rangle$ at the localized site, while $\alpha$ and $\beta_{i k}$ are variational parameters. From the resulting optimized energy $E$, we can define the stabilization energy as $T_{K}^{*}=E_{1}-E$. The Kondo energy scale defined in this way becomes

$$
T_{K}^{*}=\left\{(D+\delta) D \exp \left[\pi E_{1} /(2 \Delta)\right]+\delta^{2} / 4\right\}^{1 / 2}-\delta / 2
$$




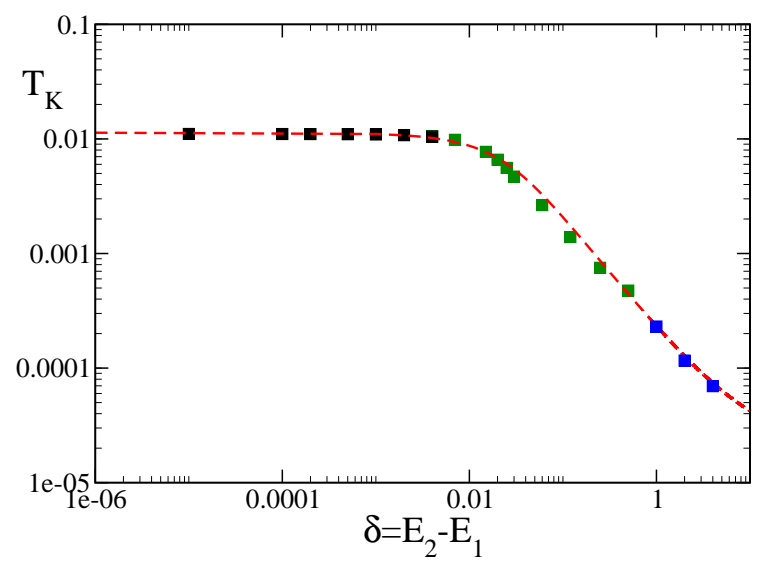

Fig. 4. Squares: Kondo energy scale determined by the width of the peak in the spectral density near the Fermi energy as a function of the splitting $\delta$. The temperatures used were $T=10^{-3}, T=10^{-4}$, and $T=10^{-5}$ depending on $\delta$ Dashed line: corresponding variational result Eq. (5) multiplied by a factor 0.606 .

This expression interpolates between the $\mathrm{SU}(4)$ result $\left(T_{K}^{*}=D \exp \left[\pi E_{1} /(4 \Delta)\right]\right.$ for $\delta=0)$ and the $\mathrm{SU}(2)$ one for one doublet only $\left(T_{K}^{*}=D \exp \left[\pi E_{1} /(2 \Delta)\right]\right.$ for $\delta \rightarrow+\infty)$.

\section{Summary and discussion}

We have studied an impurity Anderson model containing two doublets, which interpolates between the cases for one level with $\mathrm{SU}(4)$ and $\mathrm{SU}(2)$ symmetry, and is of interest for several nanoscopic systems, using the non-crossing approximation (NCA).

We have shown that the NCA provides reasonable results for the equilibrium spectral density. The values of the spectral density for both doublets agree within $20 \%$ with the predictions of the Friedel sum rule, in spite of the fact that it is not expected to satisfy Fermi liquid relationships at zero temperature.

In addition, the Kondo temperature scale $T_{K}$ obtained from the width of the peak in spectral density near the Fermi energy agrees very well with the stabilization energy of a variational calculation, in spite of the change in several orders of magnitude of $T_{K}$ when the splitting between both doublets is changed.

The approach seems promising for studying transport properties within a nonequilibrium formalism. Work in this direction is in progress. 


\section{Acknowledgments}

One of us (A. A. A.) is partially supported by CONICET, Argentina. This work was partially supported by PIP No 11220080101821 of CONICET, and PICT Nos 2006/483 and R1776 of the ANPCyT.

\section{References}

[1] L. P. Kouwenhoven and L. I. Glazman, Phys. World 14, 33 (2001).

[2] A. A. Aligia and A. M. Lobos, J. Phys.: Condens. Matter 17, S1095-S1122 (2005).

[3] A. A. Aligia, Phys. Rev. Lett. 96, 096804 (2006).

[4] J. Nygard, D. Cobden, and P. Lindelhof, Nature (London) 408, 342 (2000).

[5] P. Jarillo-Herrero, J. Kong, H. S. J. van der Zant, C. Dekker, L. P. Kouwenhoven, and S. De Franceschi, Nature (London) 434, 484 (2005).

[6] A. Makarovski, J. Liu, and G. Finkelstein, Phys. Rev. Lett. 99, 066801 (2007).

[7] J. S. Lim, M. S. Choi, M. Y. Choi, R. Lopez, and R. Aguado, Phys. Rev. B 74, 205119 (2006).

[8] C. A. Büsser and G. B. Martins, Phys. Rev. B 75, 045406 (2007).

[9] F. B. Anders, D. E. Logan, M. R. Galpin, and G. Finkelstein, Phys. Rev. Lett. 100, 086809 (2008).

[10] K. Hallberg, A. A. Aligia, A. P. Kampf, and B. Normand, Phys. Rev. Lett. 93, 067203 (2004).

[11] J. Rincón, A. A. Aligia, and K. Hallberg, Phys. Rev. B 79, 035112 (2009).

[12] A. M. Lobos and A. A. Aligia, Phys. Rev. Lett. 100, 016803 (2008).

[13] G. Begemann, D. Darau, A. Donarini, and M, Grifoni, Phys. Rev. B 77, 201406(R) (2008).

[14] J. Rincón, K. Hallberg, A. A. Aligia, and S. Ramasesha, Phys. Rev. Lett. 103, 266807 (2009).

[15] N. S. Wingreen and Y. Meir, Phys. Rev. B 49, 11040 (1994)

[16] P. Roura Bas and A. A. Aligia, J. Phys. Cond. Matt. 22, 025602 (2010).

[17] D.C. Langreth, Phys. Rev. 150, 516 (1966).

[18] Some technical details that allowed us to reach such low temperatures are briefly explained elsewhere [16].

[19] T. A. Costi, J. Kroha, and P. Wölfle, Phys. Rev. B 53, 1850 (1996) 